\title{
SOME APPLICATIONS OF INFINITARY LOGICAL LANGUAGES IN UNIVERSAL ALGEBRA
}

\author{
ALEKSANDR G. PINUS
}

\begin{abstract}
Some examples are given of applications of an infinite logical language in universal algebra, in the algebraic geometry of universal algebras, in the theory of implicit operations on algebras, in the Galois theory between automorphisms of universal algebras and its subalgebras of fixed points, in the theory of Hamiltonian closure of subalgebras and other areas.
\end{abstract}

\section{INTRODUCTION}

Historically, the first logical language is the first order language (language $L_{\omega \omega}$ ). It has a well developed model theory, which is the basis for the application of logical languages in other fields of mathematics (algebra, number theory, topology, calculus, etc.). The first examples of effective applications of $L_{\omega \omega}$ in algebra use Malcev's Compactness Theorem to obtain some local theorems in group theory: if all finitely generated subgroups of a group $G$ belong to a class of groups, then the group itself belongs to this class. There are other well-known applications of the language $L_{\omega \omega}$ in algebra such as the ultraproduct technique and model completeness in field theory (papers by I. Ax, S. Kochen and Ju. Ershov) and in number theory such as ones implying the solution of problems by Artin and Leng. Keisler-Shelah theorem on an isomorphism of some ultrapowers of elementary equivalent models has many applications in algebra.

This set of examples of applications of the language $L_{\omega \omega}$ in algebra can be extended easily (see details, e.g., in $[2,5]$ ). Henceforth, we consider algebraic systems of not larger than countable signature.

At the same time with the development of the language $L_{\omega \omega}$, some interest for other (more strong in the sense of expressivity) logical languages appears. Such languages are natural and not very natural generalizations of the language $L_{\omega \omega}$, e.g., languages with infinitely long formulas ( languages of the form $L_{k \lambda}$ for the cardinals $k, \lambda$ greater than $\omega$ ), languages with generalized quantifiers, second order language and its fragments and so on (see [1] for details on these languages and their model theories).

Because of the larger expressivity of these languages, their model theories are poorer than those of the language $L_{\omega \omega}$. One of the fundamental restrictions here is the following Lindstrom theorem.

$M S C$ (2010): primary 08-02.

Keywords: infinite logical language, universal algebra, geometrical equivalence of algebras, Galois theory, Hamiltonian algebras. 
Theorem $1.1([7])$. Let the logical language $L$ be closed for the logical connectives $\wedge, \neg$ and the quantifier $\exists$. If the compactness and Skolem-Lowenheim theorem hold for this language, then the expressive possibilities of the language $L$ are exactly the same as those of the language $L_{\omega \omega}$.

Nevertheless, languages that are richer than $L_{\omega \omega}$ have some effective algebraic applications. The purpose of this paper is to attract attention to applications of the language $L_{\omega_{1} \omega}$ in universal algebra and give some examples of such applications.

\section{The language $L_{\omega_{1} \omega}$ AND its MOdel theory}

First, we recall the inductive (by the complexity of formulas) definition of the formulas of the language $L_{k \omega}$ with a signature $\sigma$. Here $k$ is some infinite cardinal:

(1) all atomic formulas of the first order language $L_{\omega \omega}$ of this signature are $L_{k \omega}$-formulas;

(2) if $\Phi$ and $\Psi$ are $L_{k \omega}$-formulas of signature $\sigma$, then $\neg \Phi, \Phi \vee \Psi, \Phi \wedge \Psi, \Phi \rightarrow \Psi$ are also $L_{k \omega}$-formulas;

(3) if $x$ is a free variable of an $L_{k \omega}$-formula $\Phi(x)$, then $\exists x \Phi(x), \forall x \Phi(x)$ are also $L_{k \omega}$-formulas;

(4) if $\Phi$ is a set of $L_{k \omega}$-formulas such that their powers are strictly less than $k$ and the union of their free variables is finite, then $\vee \Phi$ and $\wedge \Phi$ are also $L_{k \omega}$-formulas.

An $L_{k \omega}$-formula is positive if its inductive construction does not require a transition from $\Phi$ to $\neg \Phi$ and from $\Phi, \Psi$ to $\Phi \rightarrow \Psi$ in step (2) of the inductive definition of $L_{k \omega}$-formulas.

The verity of $L_{k \omega}$-formulas on the algebraic system $\mathfrak{A}$ is defined by the standard induction (as for the language $L_{\omega \omega}$ ) and for formulas of the form $\wedge \Phi, \vee \Phi$, it is defined as follows: $\mathfrak{A} \models \wedge \Phi(\mathfrak{A} \models \wedge \Psi)$ iff $\mathfrak{A} \models \Psi$ for all (for some) formulas $\Psi$ from $\Phi$.

Thus, any $L_{\omega \omega}$-formula is also an $L_{k \omega}$-formula and the language $L_{k \omega}$ has all expressive possibilities of the first order language $L_{\omega \omega}$.

It is easy to see that the following properties can be expressed in $L_{\omega_{1} \omega}$ : for the ordered fields being Archimedian, periodicity of groups and other important properties of the classical and universal algebras that cannot be expressed in the first order language. So, the language $L_{\omega_{1} \omega}$ has much stronger expressive possibilities than the language $L_{\omega \omega}$. From the Lindstrom theorem for the language $L_{\omega_{1} \omega}$, we obtain Compactness Theorem (for any family of formulas $T$ of the considered language, the satisfiability of any finite subfamily of the family $T$ implies the satisfiability of the family $T$ ) and the Skolem-Lowenheim theorem (any satisfiable formula of the considered language has not greater than a countable model) cannot hold simultaneously.

As the property of the finiteness of the model is expressed in the language $L_{\omega_{1} \omega}$, the compactness theorem is false for this language.

For the language $L_{\omega_{1} \omega}$, the Skolem-Lowenheim theorem holds, namely if an $L_{\omega_{1} \omega}$-formula $\Phi$ is true on some infinite algebraic system $\mathfrak{A}$, then, for any infinite cardinality not exceeding $|\mathfrak{A}|$, there exists an algebraic system such that $\Phi$ is true on it (see, e.g., [6]). However, the corresponding variant of the Tarsky theorem (for 
any $L_{\omega \omega}$-formula $\Phi$ having an infinite model, there exists a $\Phi$-model of any infinite cardinality) does not hold. Let us recall that Hanf number of any logical language $L$ is the least cardinal $h_{L}$ satisfying the following condition. If an $L$-formula $\Phi$ has a model of cardinality at least $h_{L}$, then there exist $\Phi$-models of an arbitrarily large cardinality. Thus, the Tarsky theorem states that $h_{L}=\aleph_{0}$. We also recall the definition of the cardinal $\beth_{\alpha}$ for any ordinal $\alpha$ : $\beth_{0}=\aleph_{0}, \beth_{\alpha+1}=2^{\beth_{\alpha}}$, and $\beth_{\beta}=\sup _{\gamma<\beta} \beth_{\gamma}$ for any limit ordinal $\beta$.

For $L_{\omega_{1} \omega}$ the following theorem holds.

Theorem 2.1 ([9]). The Hanf number for $L_{\omega_{1} \omega}$ is equal to $\beth_{\omega_{1}}$.

One of the most important theorems in the model theory of $L_{\omega_{1} \omega}$ is the following Scott theorem.

Theorem 2.2 ([23]). For any algebraic system $\mathfrak{A}$ of at most countable cardinality there exists an $L_{\omega_{1} \omega}$-sentence $\Phi_{\mathfrak{A}}$ (the Scott formula for $\left.\mathfrak{A}\right)$ such that, for any algebraic system $\mathfrak{B}$ of at most countable cardinality, the following conditions are equivalent:

(1) $\mathfrak{B} \models \Phi_{\mathfrak{A}}$;

(2) $\mathfrak{B} \cong \mathfrak{A}$.

We also have the following analogue of this theorem.

Theorem 2.3 ([12]). For any algebraic system $\mathfrak{A}$ of at most countable cardinality, there exists a positive $L_{\omega_{1} \omega}$-sentence $\Phi_{\mathfrak{A}}^{+}$such that for any algebraic system $\mathfrak{B}$ of at most countable cardinality the following conditions are equivalent:

(1) $\mathfrak{B} \models \Phi_{\mathfrak{A}}^{+}$;

(2) $\mathfrak{B}$ is some homomorphic image of the system $\mathfrak{A}$.

One of the strongest restrictions on the expressive possibility of $L_{\omega_{1} \omega}$ is the following result by E. Lopec-Escobar [8]. The property of being a well-ordered model is not expressible in $L_{\omega_{1} \omega}$ (no set of $L_{\omega_{1} \omega}$-formulas of signature $\langle\leqslant\rangle$ exists such that the models of this set of formulas are all well-ordered sets and only they).

Some other restrictions on the expressive possibility of $L_{\omega_{1} \omega}$ are given in the following theorems.

Theorem 2.4 ([4]). For any $L_{\omega_{1} \omega}$-sentence $\Phi$ such that $\Phi$ has exactly $k$ pairwisely nonisomorphic countable models (where $\aleph_{1} \leqslant k<2^{\aleph_{0}}$ ), it follows that $\Phi$ has an uncountable model.

Theorem 2.5 ([24]). ( $V=L)$ For any $L_{\omega_{1} \omega}$-sentence $\Phi$, such that $\Phi$ has exactly $k$ pairwisely nonisomorphic models of cardinality $\aleph_{1}$ (where $1 \leqslant k<2^{\aleph_{1}}$ ), it follows that $\Phi$ has a model of cardinality $2^{\aleph_{1}}$.

By analogy with $L_{\omega \omega}, L_{\omega_{1} \omega}$-formula is called unquantified ( $\forall$-formula, $\exists$-formula, $\forall \exists$-formula, $\exists \forall$-formula, respectively) if $\Phi$ does not contain the quantifiers $\forall, \exists$ (has the form $\forall x_{1}, \ldots, x_{n} \Psi\left(x_{1}, \ldots, x_{n}\right), \exists x_{1}, \ldots, x_{n} \Psi\left(x_{1}, \ldots, x_{n}\right), \forall x_{1}, \ldots, x_{n}$ $\exists y_{1}, \ldots, y_{m} \Psi\left(x_{1}, \ldots, x_{n}, y_{1}, \ldots y_{m}\right), \quad \exists x_{1}, \ldots, x_{n} \forall y_{1}, \ldots, y_{m} \Psi\left(x_{1}, \ldots, x_{n}, y_{1}, \ldots\right.$, $\left.y_{m}\right)$, respectively, for some unquantified formula $\left.\Psi\right)$.

For $L_{\omega_{1} \omega}$, we have some analogue of Lindon theorem (on the characterization of the positive $L_{\omega \omega}$-formulas) and of Los theorem (on the characterization of first order $\forall$-formulas). 
Theorem 2.6 ([8]). An $L_{\omega_{1} \omega}$-formula $\Phi$ is preserved by homomorphisms iff $\Phi$ is equivalent to some positive $L_{\omega_{1} \omega}$-formula.

Theorem 2.7 ([10]). An $L_{\omega_{1} \omega}$-formula $\Phi$ is preserved by transitions to subsystems iff $\Phi$ is equivalent to some $\forall$-formula of $L_{\omega_{1} \omega}$. form

A $\forall$-formula of the language $L_{\omega_{1} \omega}$ is called an $\infty$-quasiidentity if it is of the

$$
\forall x_{1}, \ldots, x_{n}\left(\bigwedge_{i \in I} \Phi_{i} \rightarrow \Psi\right),
$$

where $I$ is a finite or countable set, $\Phi_{i}(i \in I)$ and $\Psi$ are some atomic formulas. A family of algebraic systems satisfying a given family of $\infty$-quasiidetities is called an $\infty$-quasivariety.

A direct spectrum $\left\{\mathfrak{A}_{i}, \varphi_{i, j} \mid i, j \in\langle I ; \leqslant\rangle\right\}$ of universal algebras is called an embeddability spectrum if each homomorphism $\varphi_{i, j}$ is an embedding of the algebra $\mathfrak{A}_{i}$ to the algebra $\mathfrak{A}_{j}$ for any $i<j$ in updirected set $\langle I ; \leqslant\rangle$. Let $\mathcal{K}$ be a family of algebras. We denote by $\mathcal{I} \mathcal{K}(\mathcal{S K}, \mathcal{P} \mathcal{K})$ the family of the algebras isomorphic to some $\mathcal{K}$-algebra (the family of subalgebras of a $\mathcal{K}$-algebras, the family of direct products of $\mathcal{K}$-algebras $)$. Let us also denote by $\lim \mathcal{K}\left(Q_{\infty} \mathcal{K}\right)$ the family of all direct limits of embedding spectrums of $\mathcal{K}$-algebras (the least $\infty$-quasivariety containing the class $\mathcal{K}$ ).

Theorem 2.8. $[[15]]$ (a) $A$ class $\mathcal{K}$ of algebras of some fixed signature is an $\infty$-quasivariety iff $\mathcal{I} \mathcal{K} \subseteq \mathcal{K}, \mathcal{S K} \subseteq \mathcal{K}, \mathcal{P K} \subseteq \mathcal{K}$, and $\lim _{\square} \mathcal{K} \subseteq \mathcal{K}$.

(b) Let $\mathcal{K}$ be a class of algebras of some fixed signature. Then, the equation $Q_{\infty} \mathcal{K}=\lim _{\longmapsto} \operatorname{ISPK}$ holds.

For details about $L_{\omega_{1} \omega}$ and its model theory see, e.g, [3, 6, 13].

\section{Geometrically and Syntactically implicitly Equivalent algebras}

In this section, we indicate a connection between geometrically and syntactically implicit equivalences of universal algebras and the language $L_{\omega_{1} \omega}$ (more precisely, between these equivalences and $\infty$-quasiidentities ).

Let us recall some concepts of algebraic geometry of universal algebras (for details see, e.g, $[13,20])$ and the theory of implicit operations on universal algebras $[15]$.

Let $V$ be a fixed variety of universal algebras of signature $\sigma$ and $X$ be a set. By $\mathcal{F}_{V}(X)$, denote the $X$-generated $V$-free algebra. For any algebra $\mathfrak{A}=\langle A ; \sigma\rangle$ and $\mathcal{F}_{V}(X)$ (for some finite $X$ ), let us denote by $V(\mathcal{F}(X) ; \mathfrak{A})$ the family of all homomorphisms from $\mathcal{F}_{V}(X)$ to $\mathfrak{A}$. Identify the homomorphism $\mu \in V(\mathcal{F}(X) ; \mathfrak{A})$ with the $n$-tuple $\bar{a}=\left\langle\mu\left(x_{1}\right), \ldots, \mu\left(x_{n}\right)\right\rangle$ of the elements of $\mathfrak{A}$ (here $X=\left\{x_{0}, \ldots, x_{n}\right\}$ ). So, $V\left(\mathcal{F}_{V}(X) ; \mathfrak{A}\right)=A^{n}$.

Let

$$
T=\left\{s_{i}\left(\bar{x}_{i}\right)=t_{i}\left(\bar{y}_{i}\right) \mid i \in I\right\}
$$

be a system of equations, where $\bar{x}_{i}, \bar{y}_{i} \subseteq X$ and $s_{i}, t_{i}$ are terms of the signature $\sigma\left(T \subseteq \mathcal{F}_{V}(X) \times \mathcal{F}_{V}(X)\right)$. The point $\mu \in A^{n}\left(\mu: \mathcal{F}_{V}(X) \rightarrow \mathfrak{A}\right)$ is a solution 
of the system $T$ in $\mathfrak{A}$ iff $\mu\left(s_{i}\left(\bar{x}_{i}\right)\right)=s_{i}^{\mathfrak{A}}\left(\mu\left(\bar{x}_{i}\right)\right)=t_{i}^{\mathfrak{A}}\left(\mu\left(\bar{y}_{i}\right)\right)=\mu\left(t_{i}\left(\bar{y}_{i}\right)\right)$ for all $s_{i}\left(\bar{x}_{i}\right)=t_{i}\left(\bar{y}_{i}\right) \in T$, i.e. iff $T \subseteq \operatorname{ker} \mu$.

For any $B \subseteq V\left(\mathcal{F}_{V}(X) ; \mathfrak{A}\right)=A^{n}$, let $B^{\prime}$ be the binary relation on $\mathcal{F}_{V}(X)$ (i.e., $\left.B^{\prime} \subseteq \mathcal{F}_{V}(X) \times \mathcal{F}_{V}(X)\right)$ such that $B^{\prime}=\bigcap_{\mu \in B} \operatorname{ker} \mu$.

For any $T \subseteq \mathcal{F}_{V}(X) \times \mathcal{F}_{V}(X)$, let $T_{\mathfrak{A}}^{\prime}=\left\{\mu \mid \mu: \mathcal{F}_{V}(X) \rightarrow \mathfrak{A}, T \subseteq \operatorname{ker} \mu\right\}$.

Then, we have the Galois-correspondence between the family of binary relations $T$ on $\mathcal{F}_{V}(X)$ and the sets $B$ of points of the space $A^{n}=V\left(\mathcal{F}_{V}(X) ; \mathfrak{A}\right)$, where $n=|X|$. The congruence $T$ of the algebra $\mathcal{F}_{V}(X)$ is $\mathfrak{A}$-closed iff $T=B^{\prime}$ for some $B \subseteq V\left(\mathcal{F}_{V}(X) ; \mathfrak{A}\right)$. The set $B \subseteq V\left(\mathcal{F}_{V}(X) ; \mathfrak{A}\right)$ is $\mathfrak{A}$-closed or an algebraic set of the space $A^{n}=V\left(\mathcal{F}_{V}(X) ; \mathfrak{A}\right)$ if $B=T_{\mathfrak{A}}^{\prime}$ for some $T \subseteq \mathcal{F}_{V}(n) \times \mathcal{F}_{V}(n)$.

This Galois-correspondence induces the following closure operations. $B \rightarrow$ $B_{\mathfrak{A}}^{\prime \prime}=\left(B^{\prime}\right)_{\mathfrak{A}}^{\prime}$ and $T \rightarrow T_{\mathfrak{A}}^{\prime \prime}=\left(T_{\mathfrak{A}}^{\prime}\right)^{\prime}$ on the subsets of the space $A^{n}=V\left(\mathcal{F}_{V}(n) ; \mathfrak{A}\right)$ and on the lattice $\operatorname{Con} \mathcal{F}_{V}(n)$ of congruences of the algebra $\mathcal{F}_{V}(n)$.

$V$-algebras $\mathfrak{A}_{1}$ and $\mathfrak{A}_{2}$ are geometrically equivalent $\left(\mathfrak{A}_{1} \stackrel{\Delta}{\sim} \mathfrak{A}_{2}\right)$ iff $T_{\mathfrak{A}_{1}}^{\prime \prime}=T_{\mathfrak{A}_{2}}^{\prime \prime}$ for any binary relation $T$ on the algebra $\mathcal{F}_{V}(n)$ for any natural $n$. It means that $\mathfrak{A}_{1} \stackrel{\Delta}{\sim} \mathfrak{A}_{2}$ iff the following condition holds. Any two systems (finite or infinite) of termal equations define the same algebraic sets on the algebra $\mathfrak{A}_{1}$ if and only if these two systems of equations define the same algebraic sets on the algebra $\mathfrak{A}_{2}$.

We have the following theorem.

Theorem 3.1 ([13]). For any $V$-algebras $\mathfrak{A}_{1}$ and $\mathfrak{A}_{2}$ the following conditions are equivalent:

(1) algebras $\mathfrak{A}_{1}$ and $\mathfrak{A}_{2}$ are geometrically equivalent;

(2) any finitely generated subalgebra of $\mathfrak{A}_{1}\left(\mathfrak{A}_{2}\right)$ can be embedded isomorphically to a direct power of $\mathfrak{A}_{2}\left(\mathfrak{A}_{1}\right)$;

(3) an $\infty$-quasiidentity holds in $\mathfrak{A}_{1}$ iff it holds in $\mathfrak{A}_{2}$.

Let us recall that an inner homomorphism (isomorphism) of a universal algebra $\mathfrak{A}$ is any homomorphism (isomorphism) between some subalgebras of this algebra. The function $f\left(x_{1}, \ldots, x_{n}\right)$ defined on the basic set of the algebra $\mathfrak{A}$ is an implicit (abstract) operation of the algebra $\mathfrak{A}$ if all subalgebras of $\mathfrak{A}$ are closed with respect to $f$ and $f$ commutes with all inner homomorphisms (isomorphisms) of $\mathfrak{A}$.

Let $\mathfrak{A}=\langle A ; \sigma\rangle$ be a universal algebra and $\bar{a}=\left\langle a_{1}, \ldots a_{n}\right\rangle$ any $n$-tuple of its elements. Denote by $D_{\bar{a}}^{+}(\bar{x})$ the positive diagram of the algebra $\langle\bar{a}\rangle_{\mathfrak{A}}$ (i.e. of the subalgebra of $\mathfrak{A}$ generated by the set $\left.\left\{a_{1}, \ldots, a_{n}\right\}\right)$ such that $\mathfrak{A} \models D_{\bar{a}}^{+}(\bar{a})$. Let $T_{\mathfrak{A}}^{n}$ be the set of all types of isomorphisms for algebras of the form $\left\langle\langle\bar{a}\rangle_{\mathfrak{A}}, a_{1}, \ldots, a_{n}\right\rangle$ (the algebras $\langle\bar{a}\rangle_{\mathfrak{A}}$ enriched by the constants $a_{1}, \ldots, a_{n}$ for any $a_{1}, \ldots, a_{n} \in \mathfrak{A}$. Let also $\operatorname{Tr}_{\sigma}^{n}$ be the set of all $n$-ary terms of the signature $\sigma$. Finally, let $\varphi: T_{\mathfrak{A}}^{n} \rightarrow \operatorname{Tr}_{\sigma}^{n}$ be a map such that for any $\bar{a}=\left\langle a_{1}, \ldots a_{n}\right\rangle, \bar{b}=\left\langle b_{1}, \ldots b_{n}\right\rangle \in A^{n}$, we have

$$
\mathfrak{A}=D_{\bar{a}}^{+}\left(b_{1}, \ldots, b_{n}\right) \rightarrow \varphi\left(\left\langle\langle\bar{a}\rangle_{\mathfrak{A}}, \bar{a}\right\rangle\right)\left(b_{1}, \ldots, b_{n}\right)=\varphi\left(\left\langle\langle\bar{b}\rangle_{\mathfrak{A}}, \bar{b}\right\rangle\right)\left(b_{1}, \ldots, b_{n}\right) .
$$

An $\infty$-positive conditional term $t_{\varphi}\left(x_{1}, \ldots, x_{n}\right)$ of the algebra $\mathfrak{A}$ is the following schema

$$
t_{\varphi}\left(x_{1}, \ldots, x_{n}\right)=\bigwedge_{\left\langle\langle\bar{a}\rangle_{\mathfrak{A}}, \bar{a}\right\rangle \in T_{\mathfrak{l}}^{n}}\left(D_{\bar{a}}^{+}\left(x_{1}, \ldots, x_{n}\right) \rightarrow \varphi\left(\left\langle\langle\bar{a}\rangle_{\mathfrak{A}}, \bar{a}\right\rangle\right)\left(x_{1}, \ldots x_{n}\right)\right)
$$


for any map $\varphi: T_{\mathfrak{A}}^{n} \rightarrow \operatorname{Tr}_{\sigma}^{n}$ such that (3.1) holds.

Every $\infty$-positive conditional term $t_{\varphi}$ defines an $\infty$-positive conditional termal function $t_{\varphi}^{\mathfrak{A}}$ on the basic set of $\mathfrak{A}$ as follows.

$$
t_{\varphi}^{\mathfrak{A}}\left(a_{1}, \ldots, a_{n}\right)=\varphi\left(\left\langle\langle\bar{a}\rangle_{\mathfrak{A}}, \bar{a}\right\rangle\right)\left(a_{1}, \ldots a_{n}\right)
$$

for any $a_{1}, \ldots, a_{n} \in \mathfrak{A}$. Every implicit operation on $\mathfrak{A}$ is a $\infty$-positive conditional termal function for $\mathfrak{A}$ and vice versa.

Algebras $\mathfrak{A}=\langle A ; \sigma\rangle$ and $\mathfrak{B}=\langle B ; \sigma\rangle$ are syntactically implicitly equivalent if the following conditions are satisfied:

(1) every $\infty$-positive conditional term of $\mathfrak{A}$ is an $\infty$-positive conditional term of $\mathfrak{B}$ and vice versa;

(2) every two $\infty$-positive conditional terms of $\mathfrak{A}$ define the same implicit operations on $\mathfrak{A}$ iff these two terms define the same operations on $\mathfrak{B}$.

The connection between syntactically implicit equivalence and the language $L_{\omega_{1} \omega}$ is given in the following theorem:

Theorem 3.2 ([14]). For any algebras $\mathfrak{A}$ and $\mathfrak{B}$, the following conditions are equivalent:

(1) the algebras $\mathfrak{A}$ and $\mathfrak{B}$ are syntactically implicitly equivalent;

(2) if an $\infty$-quasyidentity is true on the algebra $\mathfrak{A}(\mathfrak{B})$, then it is also true on $\mathfrak{B}(\mathfrak{A})$.

Theorems 2.8 and 3.1 imply an equality of the geometrically and syntactically implicit equivalences of algebras.

From this theorems we also obtain

Corollary 3.3 ([13, 14]). For any infinite algebra $\mathfrak{A}$ and each cardinal $k$ such that $2^{\aleph_{0}} \leqslant k \leqslant|\mathfrak{A}|$ there exists a subalgebra $\mathfrak{B}$ of the algebra $\mathfrak{A}$ such that the cardinality of $\mathfrak{B}$ is $k$ and this subalgebra is geometricaly (syntactically implicitly) equivalent to the algebra $\mathfrak{A}$.

The restriction $2^{\aleph_{0}} \leqslant k$ in this corollary is essential.

By analogy with the concept of rational equivalence of algebras, let us say that the algebras $\mathfrak{A}=\left\langle A ; \sigma_{1}\right\rangle$ and $\mathfrak{B}=\left\langle B ; \sigma_{2}\right\rangle$ of signatures $\sigma_{1}$ and $\sigma_{2}$ respectively, are implicitly (abstractly) equivalent if there exists a bijection $\varphi$ of $A$ onto $B$ such that $\varphi$-conjugates of basic operations of $\mathfrak{B}$ are implicit (abstract)operations of $\mathfrak{A}$ and $\varphi^{-1}$-conjugates of basic operations of $\mathfrak{A}$ are implicit (abstract) operations of $\mathfrak{B}$. In other words, the algebras $\mathfrak{A}$ and $\mathfrak{B}$ are implicitly (abstractly) equivalent if $\varphi^{-1} \mathrm{IO}(\mathfrak{B}) \varphi=\operatorname{IO}(\mathfrak{A})\left(\varphi^{-1} \mathrm{AO}(\mathfrak{B}) \varphi=\mathrm{AO}(\mathfrak{A})\right)$, where $\operatorname{IO}(\mathfrak{A})(\mathrm{AO}(\mathfrak{A}))$ is the family of all implicit (abstract) operations of the algebra $\mathfrak{A}$.

Let $\pi$ be a permutation of symbols of signature $\sigma$ such that for $f \in \sigma$ the arities of $f$ and $\pi(f)$ coincide. For any algebra $\mathfrak{A}=\langle A ; \sigma\rangle$, denote by $\mathfrak{A}^{\pi}$ the algebra of signature $\sigma$ with the same basic set and such that $f_{i}^{\mathfrak{A}^{\pi}}=f_{\pi(i)}^{\mathfrak{A}}$.

The algebra $\mathfrak{A}=\langle A ; \sigma\rangle$ is called implicitly (abstractly ) categorical if for, any algebra $\mathfrak{B}=\langle B ; \sigma\rangle$, the implicit (abstract) equivalence of $\mathfrak{A}$ and $\mathfrak{B}$ (via bijection $\varphi: A \rightarrow B$ ) implies that there exists $\pi$ such that $\varphi$ is an isomorphism of $\mathfrak{A}^{\pi}$ onto $\mathfrak{B}$.

So, we have 
Theorem 3.4 ([18]). The classes of all implicitly (abstractly) categorical algebras of any fixed signatures are axiomatizable by some $\forall \exists-(\exists \forall-)$ formulas of the language $L_{\omega_{1} \omega}$.

Corollary 3.5 ([18]). a) The classes of implicitly (abstractly) categorical algebras of some fixed signatures are closed with respect to increasing chains.

b) For any implicitly (abstractly) categorical algebra $\mathfrak{A}$ there exists its finite or countable subalgebra $\mathfrak{A}^{\prime}$ such that all subalgebras $\mathfrak{A}^{\prime \prime}$ between $\mathfrak{A}^{\prime}$ and $\mathfrak{A}$ are implicitly (abstractly) categorical.

\section{The language $L_{\omega_{1} \omega}$ And Automorphisms of Algebras}

In this section, we give some examples of applications of the language $L_{\omega_{1} \omega}$ in the theory of automorphisms of universal algebras.

The problem of a connection between automorphisms of algebraic systems and the sets of their fixed points is one of the classical algebraic problems and the first result obtained in this area is Galois theory of the fields. Results concerning this connection in more general case (namely, for universal algebras) can be found in a monograph by B. I. Plotkin [21].

By $\operatorname{Sub} \mathfrak{A}$ (Aut $\mathfrak{A}$ ) denote the lattice of subalgebras (the group of automorphisms) of the universal algebra $\mathfrak{A}$. Let $f \in$ Aut $\mathfrak{A}$. By fix $f$ denote a subalgebra $\{a \in \mathfrak{A} \mid f(a)=a\}$ of $\mathfrak{A}$. Finally, if $\mathfrak{B} \in \operatorname{Sub} \mathfrak{A}$ then by $\operatorname{Stab}(\mathfrak{B})$ denote the subgroup $\{f \in$ Aut $\mathfrak{A} \mid f(b)=b$ for any $b \in \mathfrak{B}\}$ of Aut $\mathfrak{A}$. We have the following Galois correspondence [22]:

$$
\begin{aligned}
& \text { Sub : Sub } \mathfrak{A} \rightarrow \operatorname{Sub} \text { Aut } \mathfrak{A}, \\
& \text { Fix : Sub Aut } \mathfrak{A} \rightarrow \operatorname{Sub} \mathfrak{A},
\end{aligned}
$$

where Fix $\mathfrak{C}=\bigcap_{f \in \mathfrak{C}} \operatorname{fix}(f)$ for $\mathfrak{C} \in \operatorname{Sub}$ Aut $\mathfrak{A}$. The maps Sub and Fix define the closure operation $\mathfrak{B} \rightarrow \overline{\mathfrak{B}}$ on the lattice $\operatorname{Sub} A$. By $f \uparrow \mathfrak{C}$, we denote the restriction of the automorphism $f$ onto a to the subalgebra $\mathfrak{C}$. So, for any $f, g \in$ Aut $\mathfrak{A}$ and any $\mathfrak{B} \in \operatorname{Sub} \mathfrak{A}$, we have $f \uparrow \mathfrak{B}=g \uparrow \mathfrak{B}$ is equivalent to $f \uparrow \overline{\mathfrak{B}}=g \uparrow \overline{\mathfrak{B}}$. Let $B$ be an arbitrary subset of the basic set $A$ of an algebra $\mathfrak{A}$. By $\mathfrak{A}_{B}$ we denote the constant enrichment of $\mathfrak{A}$ to the signature $\sigma_{B}=\sigma \cup\left\langle c_{b} \mid b \in B\right\rangle$ such that the interpretation of the constant $c_{b}$ in $\mathfrak{A}_{B}$ is $b$.

By the Scott theorem, we obtain the following description of $\overline{\mathfrak{B}}$ for any $\mathfrak{B} \in$ Sub $\mathfrak{A}$.

Theorem 4.1 ([16]). Let $\mathfrak{A}=\langle A ; \sigma\rangle$ be a finite or countable algebra and let $\mathfrak{B}=\langle B ; \sigma\rangle$ be its subalgebra. The element $d$ in $A$ belongs to $\overline{\mathfrak{B}}$ iff there exists some $L_{\omega_{1} \omega}$-formula $\Phi_{d}(x)$ of signature $\sigma_{B}$ such that $\mathfrak{A}_{B}=\exists ! x \Phi_{d}(x)$ and $\mathfrak{A}_{B}=\Phi_{d}(d)$ (if $\mathfrak{A}$ is finite, then the formula $\Phi_{d}(x)$ exists in the language $\left.L_{\omega \omega}\right)$.

Here $\mathfrak{A}_{B} \models \exists ! x \Phi(x)$ means that there exists a unique $x$ such that $\Phi(x)$ holds.

We say that a subalgebra $\mathfrak{B}$ of the algebra $\mathfrak{A}$ is Galois-closed, if $\overline{\mathfrak{B}}=\mathfrak{B}$. We also say that a subalgebra $\mathfrak{B}=\langle B ; \sigma\rangle$ of the algebra $\mathfrak{A}$ a $\exists ! L_{\omega_{1} \omega}$-subalgebra of $\mathfrak{A}$ if for any $L_{\omega_{1} \omega}$-formula $\Phi(x)$ of signature $\sigma_{B}$ and any element $d \in \mathfrak{A}$, the conditions $\mathfrak{A}_{B} \models \exists ! x \Phi(x)$ and $\mathfrak{A}_{B} \models \Phi(d)$ imply $d \in B$.

Thus, we have 
Corollary $4.2([16])$. For a subalgebra $\mathfrak{B}$ of an algebra $\mathfrak{A}$ the following conditions are equivalent:

(1) $\mathfrak{B}$ is Galois-closed;

(2) $\mathfrak{B}$ a $\exists ! L_{\omega_{1} \omega}$-subalgebra of $\mathfrak{A}$.

Let us recall, that the algebra $\mathfrak{A}$ is rigid (epirigid) if all authomorphisms (endomorphisms of $\mathfrak{A}$ on itself ) of $\mathfrak{A}$ are trivial. The algebra $\mathfrak{A}$ is $n$-transitive if, for any $a_{1}, \ldots, a_{n}, b_{1}, \ldots, b_{n} \in \mathfrak{A}$ such that $a_{i}=a_{j}$ iff $b_{i}=b_{j}(i, j \leqslant n)$, there exists an automorphism $\varphi$ of the algebra $\mathfrak{A}$ such that $\varphi\left(a_{i}\right)=b_{i}(i \leqslant n)$. From the Scott theorem (Theorem 2.2) and Theorem 2.3, we obtain that the property of countable algebras being rigid (epirigid, $n$-transitive) can be expressed in the language $L_{\omega_{1} \omega}$.

A function $f\left(x_{1}, \ldots, x_{n}\right)$ on the basic set $A$ of some universal algebra $\mathfrak{A}$ is called $L_{\omega_{1} \omega}$-definable $\left(L_{\omega_{1} \omega^{\prime}}^{+}\right.$-definable) if there exists an $L_{\omega_{1} \omega}$-formula $\left(L_{\omega_{1} \omega^{-}}^{+}\right.$-formula) $\Phi\left(x_{1}, \ldots, x_{n}, y\right)$ of signature $\sigma$ such that, for any $a_{1}, \ldots, a_{n}, b \in A$, the following conditions are equivalent:

(1) $\mathfrak{A}=\Phi\left(a_{1}, \ldots a_{n}, b\right)$;

(2) $b=f\left(a_{1}, \ldots, a_{n}\right)$.

We have

Theorem 4.3 ([12]). For any finite or countable universal algebra $\mathfrak{A}=\langle A ; \sigma\rangle$ and any function $f\left(x_{1}, \ldots, x_{n}\right)$ on $A$, the following conditions are equivalent:

(1) $f\left(x_{1}, \ldots, x_{n}\right)$ is $L_{\omega_{1} \omega}$-definable $\left(L_{\omega_{1} \omega}^{+}\right.$-definable $)$;

(2) $f\left(x_{1}, \ldots, x_{n}\right)$ commutes with all automorphisms of the algebra $\mathfrak{A}$ (with all endomorphisms of the algebra $\mathfrak{A}$ on itself).

Algebras $\mathfrak{A}=\left\langle A ; \sigma_{1}\right\rangle$ and $\mathfrak{B}=\left\langle B ; \sigma_{2}\right\rangle$ are called $L_{\omega_{1} \omega^{-}}$equivalent $\left(L_{\omega_{1} \omega^{-}}^{+}\right.$ equivalent) if there exists a bijection $\eta$ from $A$ on $B$ such that this bijection conjugates the sets of $L_{\omega_{1} \omega}$-definable $\left(L_{\omega_{1} \omega}^{+}\right.$-definable) functions of the algebras $\mathfrak{A}$ and $\mathfrak{B}$. In other words, $\mathfrak{A}$ and $\mathfrak{B}$ are equivalent if, for any basic operations $f\left(x_{1}, \ldots, x_{n}\right)$ of $\mathfrak{A}$, the function $\eta f\left(\eta^{-1}\left(x_{1}\right), \ldots, \eta^{-1}\left(x_{n}\right)\right)$ is $L_{\omega_{1} \omega}$-definable (correspondingly $L_{\omega_{1} \omega^{-}}^{+}$-definable) for $\mathfrak{B}$ and vice versa: for any basic operations $g\left(x_{1}, \ldots, x_{n}\right)$ in $\mathfrak{B}$ the function $\eta^{-1} g\left(\eta\left(x_{1}\right), \ldots, \eta\left(x_{n}\right)\right)$ is $L_{\omega_{1} \omega}$-definable $\left(L_{\omega_{1} \omega^{-}}^{+}\right.$-definable) for $\mathfrak{A}$.

From Theorem 4.3 we have

Corollary 4.4 ([12]). For finite or countable algebras $\mathfrak{A}=\left\langle A ; \sigma_{1}\right\rangle$ and $\mathfrak{B}=$ $\left\langle B ; \sigma_{1}\right\rangle$, the following conditions are equivalent:

(1) $\mathfrak{A}$ and $\mathfrak{B}$ are $L_{\omega_{1} \omega}$-equivalent $\left(L_{\omega_{1} \omega}^{+}\right.$-equivalent);

(2) there exists a bijection $\eta$ from $A$ on $B$ such that $\eta$ conjugates the families of all automorphisms of $\mathfrak{A}$ and $\mathfrak{B}$ (all endomorphisms of these algebras onto themselves).

\section{The language $L_{\omega_{1} \omega}$ And the COngruences of Universal algebras}

In this section, we consider the properties of universal algebras that are connected with their congruences and are expressible in the language $L_{\omega_{1} \omega}$, for instance: simplicity, $p$-pseudosimplicity, subdirect irreducibility, being Hamiltonian and some corollaries of this expressibility. 
The base of this expressivity is the well-known Malcev lemma: for any universal algebra $\mathfrak{A}=\langle A ; \sigma\rangle$ and for any $a, b, c, d \in A$, the following conditions are equivalent:

(1) $\langle a, b\rangle \in \theta_{c, d}^{\mathfrak{A}}$ (the principal congruence of the algebra $\mathfrak{A}$ generated by the pair $\langle c, d\rangle)$;

(2) for some natural $n$, there exist terms $p_{0}\left(x, \bar{y}_{1}\right), \ldots, p_{n}\left(x, \bar{y}_{n}\right)$ of signature $\sigma$ and $n$-tuples $\bar{e}_{i}$ of the elements in $A$ such that $a=p_{0}\left(c, \bar{e}_{1}\right), p_{i}\left(d, \bar{e}_{i}\right)=$ $p_{i+1}\left(c, \bar{e}_{i+1}\right)$ for $i \leqslant n$ and $p_{n}\left(d, \bar{e}_{n}\right)=b$.

Thus, for any signature $\sigma$, there exists an $\exists$-formula $\Phi_{\text {Con }}(x, y, z, u)$ of the language $L_{\omega_{1} \omega}$ such that, for any algebra $\mathfrak{A}=\langle A ; \sigma\rangle$ and any $a, b, c, d$, the following conditions are equivalent:

(1) $\langle a, b\rangle \in \theta_{c, d}^{\mathfrak{A}}$;

(2) $\mathfrak{A}=\Phi_{\text {Con }}(a, b, c, d)$.

From this statement we obtain that the followng properties for algebras can be expressed by $L_{\omega_{1} \omega}$-formulas: simplicity, subdirect irreducibility, $p$-pseudosimplicity for at most countable algebras.

By this and by Theorems 2.4 and 2.5 we obtain

Corollary 5.1 ([17]). (a) Let $V$ be a variety of universal algebras such that $V$ has exactly $k$ pairwisely nonisomorphic countable simple (subdirectly irreducible) algebras (for $\aleph_{1} \leqslant k<2^{\aleph_{0}}$ ). Then $V$ contains an uncountable simple (subdirectly irreducible) algebra.

(b) $(V=L)$ Let $V$ be a variety of universal algebras such that $V$ has exactly $k$ pairwisely nonisomorphic simple (subdirectly irreducible) algebras of cardinality $\aleph_{1}\left(\right.$ for $\left.1 \leqslant k<2^{\aleph_{1}}\right)$. Then $V$ contains a simple (subdirectly irreducible) algebra of cardinality $2^{\aleph_{1}}$.

In [11] (with an assumption of GCH), it is proved that the spectrum of any simple algebra (the family of infinite powers of simple algebras) of any variety is $\varnothing$, or $\left\{\aleph_{0}\right\}$, or $\left\{\aleph_{0}, 2^{\aleph_{0}}\right\}$, or $\left[\aleph_{0}, \infty\right)$.

From this assertion and from Corollary 5.1 (b) we obtain:

Corollary $5.2(V=L)$. Let $V$ be a variety of universal algebras. If $V$ has a simple algebra of continuum pover and the number of pairwisely nonisomorphic such simple algebras in $V$ is less than $2^{\aleph_{1}}$, then $V$ has simple algebras of any infinite cardinality.

Let us recall that a universal algebra $\mathfrak{A}=\langle A ; \sigma\rangle$ is Hamiltonian if any of its subalgebras is a congruence class (the class of equivalence for some congruence of the algebra $\mathfrak{A})$.

This concept implies some natural certainty of Hamiltonian closure operation (for details see [21]) on the lattice of subalgebras Sub $\mathfrak{A}$ of the algebra $\mathfrak{A}$. Namely, for any $\mathfrak{B}=\langle B ; \sigma\rangle \in \operatorname{Sub} \mathfrak{A}$, the Hamiltonian closure $\overline{\mathfrak{B}}^{h}$ of $\mathfrak{B}$ is the smallest subalgebra of $\mathfrak{A}$ including $\mathfrak{B}$ and such that $\overline{\mathfrak{B}}^{h}$ is a congruence class of $\mathfrak{A}$. An algebra $\mathfrak{A}$ is Hamiltonian simple if, for every its subalgebra $\mathfrak{B}$ with more than one element, we have $\overline{\mathfrak{B}}^{h}=\mathfrak{A}$.

We can state the following theorem 
Theorem 5.3 ([19]). The classes of all Hamiltonian and Hamiltonian simple algebras of any fixed signature are axiomatizable by some $L_{\omega_{1} \omega}$-formulas in the class of all algebras of this signature.

Corollary $5.4([19])$. Let $\mathcal{K}$ be a class of algebras axiomatizable in the language $L_{\omega_{1} \omega}$. If there exists Hamiltonian (Hamiltonian simple) $\mathcal{K}$-algebra of some infinite power $k$ then there exists a Hamiltonian (Hamiltonian simple) $\mathcal{K}$-algebra of any infinite cardinality less than $k$. In the case $k \geqslant \beth_{\omega_{1}}$, there exist Hamiltonian (Hamiltonian simple) $\mathcal{K}$-algebras for any infinite cardinality.

\section{REFERENCES}

[1] J. Barwise and S. Feferman (eds.), Model-Theoretical Logic, North-Holland, Amsterdam, 1985.

[2] C. C. Chang and H. J. Keisler, Model Theory, North-Holland, Amsterdam, 1973.

[3] M. A. Dickman, Large Infinitary Languages. Model Theory, North-Holland, Amsterdam, 1975.

[4] V. Harnik and M. Makkai, A tree argument in infinitary model theory, Proc. Amer. Math. Soc. 67 (1977), 309-314.

[5] G. U. Jensen and H. Lenzing, Model-Theoretic Algebra, Gordon and Breach Scienc. Publ. Comp., New York, 1989.

[6] H. J. Keisler, Model Theory for Infinitary Logic, North-Holland, Amsterdam, 1971.

[7] P. Lindstrom, On extensions of elementary logic, Theoria 35 (1969), 1-11.

[8] E. Lopec-Escobar, An interpolation theorem for denumerable long sentences, Fund. Math. 57 (1965), 253-272.

[9] E. Lopez-Escobar, On definable well orderings, Fund. Math. 59 (1966), 13-21.

[10] J. Malitz, Infinite analogs of theorems from first order model theory, J. Symbol. Logic 36 (1971), 216-228.

[11] R. McKenzie and S. Shelah, The cardinals of simple models for universal theories, in: Proc. of the Tarski Symposium, Amer. Math. Soc., Providence, Rhode Island, 1974, 53-75.

[12] A. G. Pinus, Definable functions of universal algebras and definable equivalence of algebras, to appear.

[13] A. G. Pinus, Geometrical scales of varieties of algebras and quasi-identities, Siberian Adv. Math. 20 (2010), 217-222.

[14] A. G. Pinus, Implicit equivalent universal algebras, Siberian Math. J. 53 (2012), 1077-1090.

[15] A. G. Pinus, On $\infty$-quasivarieties, Russian Math. (Iz. VUZ) 55 (2011), 33-37.

[16] A. G. Pinus, On the classical Galois-closure for the universal algebras, to appear.

[17] A. G. Pinus, The properties of rigidity, pseodosimplity and transitivity of algebraic systems which are expressed in the language $L_{\omega_{1} \omega}$, to appear.

[18] A. G. Pinus, Rational equivalence of algebras, its "clon" generalizations and "clon" categoricity, to appear.

[19] A. G. Pinus, The Hamiltonian closure on universal algebras, to appear.

[20] B. I. Plotkin, Some concepts of algebraic geometry in universal algebra, Algebra and Analisis 9 (1997), 224-248.

[21] B. I. Plotkin, The Groups of Automorphisms of Algebraic Systems, Science Publ., Moscow, 1966.

[22] R. Poschel and L.A. Kaluznin, Funktionen and Relationen Algebras, Deutscher Verlag d. Wissensch., Berlin, 1978.

[23] D. Scott, Logic with denumerable long formulas and finite strings of quantifiers, in: Theory of Models, North-Holland, Amsterdam, 1965, 329-341.

[24] S. Shelah, Categoricity in $\aleph_{1}$ of sentences of $L_{\omega_{1} \omega}(Q)$, Israel J. Math. 20 (1975), 127-148. 
Aleksandr G. Pinus, Novosibirsk State Technical University, 20 Prospekt K. Marksa, Novosibirsk, 630073, Russia

e-mail: ag.pinus@gmail.com 
